\title{
Heterogeneous Autonomous Mobile Maritime Expeditionary Robots
}

\author{
Vladimir Djapic, Captain (Ret). George Galdorisi, Anthony Jones, Michael Ouimet \\ SPAWAR Systems Center Pacific (SSC Pacific) \\ San Diego, USA, \\ \{vdjapic, adjones3,ouimet\}@spawar.navy.mil, george.galdorisi@navy.mil
}

\begin{abstract}
For the ONR-funded Heterogeneous Adaptive Maritime Mobile Expeditionary Robots (HAMMER) project, we work on cooperative autonomy for a fleet of unmanned vehicles working together in the aerial, water surface, and underwater domains. Each of these systems work well independently, but our goal is to integrate their performance into one system of vehicles that can safely perform cooperative tasks. The challenges we are working on include creating reliable communications links between vehicles in the harsh low bandwidth maritime environment, integrating novel onboard sensors and inter-vehicle communication to create filters to estimate the state of the network, and creating autonomous takeoff-and-landing algorithms between the aerial/underwater vehicles and the surface "mothership" vehicle. The surface vehicle is envisioned to be capable of transporting the aerial and underwater vehicles as well as providing mission-lengthening power. Possible applications of this system include automated deployment and recovery of data-collecting unmanned underwater vehicles and an ad hoc wireless network where the aerial vehicle relays timesensitive data collected from the surface or underwater vehicle to a human on a ship many miles away. In a separate but related project, we are also determining human-autonomy teaming required for future Naval programs, assessing the state-of-the-art algorithms, and creating open challenge problems to academia to fill gaps based on the Navy's need.
\end{abstract}

Keywords-autonomous, mobile, robots, cooperative controls

\section{Perspective}

In his best-selling book, War Made New, military historian Max Boot supports his thesis with historical examples to show how technological-driven "Revolutions in Military Affairs" have transformed warfare and altered the course of history $[1,2]$. The U.S. military has embraced a wave of technological change that has constituted a true revolution in military affairs.

Unquestionably, one of the most rapidly growing areas of military technology adoption involves unmanned systems. In the past decade the military's use of unmanned aerial vehicles (UAVs) has increased from only a handful to more than 10,000, while the use of unmanned ground vehicles (UGVs) has exploded from zero to more than 12,000. The use of unmanned surface vehicles (USVs) and unmanned underwater vehicles (UUVs) is also growing as USVs and
UUVs are proving to be increasingly useful for a variety of military applications.

The expanding use of unmanned systems - and especially armed, unmanned systems - is not only changing the face of modern warfare, but is also altering the process of decision-making in combat operations. Indeed, it has been argued that the rise in drone warfare is changing the way we conceive of and define "warfare" itself. These systems have been used extensively in the conflicts in Iraq and Afghanistan, and will continue to be equally relevant-if not more so-as the United States' strategic focus shifts towards the Asia-Pacific region and the high-end warfare this strategy requires. The exploding use of unmanned systems (UxS) is already creating strategic, operational, and tactical possibilities that did not exist a decade ago.

However, while these unmanned systems are of enormous value today and are evolving to deliver better capabilities to the warfighter, it is their promise for the future that causes the most excitement. Indeed, these systems have created a substantial buzz in policy, military, industry and academic circles. One of the most cutting-edge - and challenging aspects of autonomous systems is having systems that operate in different domains: Unmanned Aerial Vehicles (UAVs), Unmanned Surface Vehicles (USVs), and Unmanned Underwater Vehicles (UUVs), work together as a heterogeneous whole.

\section{The U. S. NAVy'S TeChNology Challenges}

The U.S. Navy aims to create multiple unmanned vehicle systems containing Unmanned Surface Vehicles (USVs), Unmanned Aerial Vehicles (UAVs), and Unmanned Underwater Vehicles (UUVs) in order to help ensure security and prosperity on the global commons. Until recent developments in technology, having USV, UAVs, and UUVs work together has only been a vision for the future. Today, scientists and engineers at the Navy's Space and Naval Warfare Systems Center Pacific (SSC Pacific) are leading an international consortium from academia, government and industry to make this vision a reality. Over the next several years, the Center for Innovative Naval TechnologiesInformation Dominance (CINT-ID) Heterogeneous Autonomous Mobile Maritime Expeditionary Robots 
(HAMMER) project, funded by Office of Naval Research (ONR), aims to successfully integrate unmanned surface, aerial, and underwater vehicles.

The HAMMER project addresses important command, control, communications, computers, intelligence, surveillance and reconnaissance (C4ISR) considerations for operationalizing a combination of UxSs, using a system-ofsystems approach. The team uses a Wave Adaptive Modular Vessel (WAM-V)-a sixteen foot catamaran USV-as the central node and main transport mechanism. The WAM-V is designed to carry UAVs and UUVs across distances greater than 100 miles and operate for months at a time. Over the next three to five years, the HAMMER project will continue to successfully integrate unmanned surface, aerial, and underwater vehicles. With the WAM-V as the vehicle, the other important parts of the HAMMER project include:

- Sensors: Some sensors are crucial to basic USV functionality while others can be attached or removed to accommodate a specific mission. Sensors are either installed directly to the payload platform of the WAM$\mathrm{V}$, or attached to a gimbal-pole system that can lower into the water. An Inertial Measurement Unit (IMU) and Global Positioning System (GPS) are crucial sensors needed for most robotic platforms.

- User Interface: Using pre-existing software and prototyping programs, a framework has been developed to integrate, collaborate, control, and interact with this project. This incorporates a publish-subscribe framework for communications and control, as well as a mission control graphical user interface (GUI) that allows users to report and interact with the HAMMER system.

- UAS: The Unmanned Aerial System (UAS) is comprised of a UAS, a payload, and the necessary onboard sensors and computer equipment. The current design of the UAS involves a helicopter vehicle with onboard autopilot and power system, as well as available space for ten pounds of payload. Currently, research is focused on core tasks for the UAS, such as takeoff and landing on the USV.

- UUVs: The HAMMER project is now testing a number of UUVs for incorporation with the WAM-V sea frame.

With the propulsion, power management, and sensor systems of the USV, the landing and takeoff algorithms of the UAV, the future integration of a UUV, as well as overarching communications framework, the HAMMER project will soon achieve heterogeneous system autonomy. Several sub-systems working together as one will minimize operator requirements and reduce the manpower necessary for system success in order to achieve true information dominance in the maritime domain.

\section{REDUCING MANPOWER COSTS}

Whether it takes two, four, six or some higher multiple of people to support one unmanned aerial system, as in the case of UAVs flying in Afghanistan that are operated from a base in Nevada, the "tail" is often obscured. When the UAV operator or technician finishes a shift, he or she goes home. This is not the current Concept of Operations (CONOPS) for unmanned aerial and maritime systems deployed from Navy ships or submarines. Currently, every operator and technician must be deployed on the ship. Each person has a bunk, must be fed, generates administrative and overhead requirements, and has quality-of-life needs that must be met. This, in turn, generates additional manpower needs that add weight and space to the ship.

This situation is exacerbated by the indisputable fact that the biggest and most rapidly-rising cost of ships and systems is manpower, which makes up close to $70 \%$ of the total ownership cost of ships [3]. This massive, manpowerinduced portion of Total Operating Cost (TOC) has the full attention of the highest levels of Navy leadership. For unmanned systems to reach their full potential, important command, control, communications, computers, intelligence, surveillance, and reconnaissance (C4ISR) considerations must be addressed. Therefore, our focus is on creating a platform in which one operator can command multiple unmanned systems. Additionally, this platform combines aerial, surface, and underwater capabilities to create a comprehensive C4ISR system that can be used in many different scenarios.

The HAMMER system will be capable of coordinating a group of multi-domain maritime autonomous systems (MAS) gathering data from the ocean over several months and will be able to grow capabilities to track and sample dynamic features. This concept was introduced with the Autonomous Ocean Sampling Network (AOSN) two decades ago [4]. The design of our system is modular, which enables flexibility and the opportunity for the cooperation of many different systems. Whether it's one UxV, one HAMMER system, or multiple HAMMER systems, they will be equipped with the capabilities and autonomy to work together seamlessly and effectively.

\section{SYSTEM COMPONENTS AND USV SENSORS}

The WAM-V's unique stability system of springs, shock absorbers, and ball joints allows the vessel to glide over waves while the payload tray remains relatively level. Additionally, the tray has a payload of up to 300 pounds. The WAM-V was provided as a basic structure without any propulsion system, power, or sensor attachments. Because of this simple structure, the WAM-V serves as an excellent experimental platform.

A propulsion system needed to be designed and manufactured for the WAM-V. Our team selected trolling 
motors. These were chosen because there's an existing familiarity with the system, as another project at SPAWAR Systems Center Pacific (SSC Pacific) Unmanned Maritime Vehicle Laboratory uses the same propulsion system. When designing the rest of the propulsion system many factors and requirements were considered. A pod-like cylindrical shape was chosen in order to extend the hull shape. In addition to serving as an attachment location for the motors, the pods were also designed as a storage container for batteries, electronics, and other equipment. Optimization calculations and tests were conducted to ensure that the pods would float behind the WAM-V. While the pods were designed to hold up to 155 pounds of payload and still float, an adjustable hinge plate attached the pods to the WAM-V.

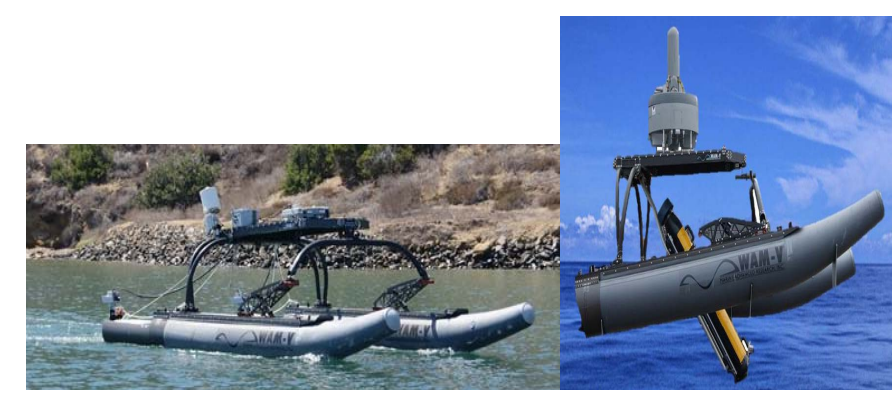

Figure 1: (left) WAM-V USV on a test mission, (right) a group of Maritime $\mathrm{UxVs}$

In order for the multiplatform implementation of the WAM-V to run the loads of the system, a range extender was implemented as the central basis of power for the surface vehicle. The concept behind a range extender can be expressed as a hybrid power system in which two sources of power are jointly used rather than one used independently. In the WAMV's particular system, Lithium-ion batteries, 16 inside each propulsion pod (chosen in order to maximize energy density and safety), were integrated with a diesel generator. The batteries have a total of 7200 watt-hours with 3600 watt-hours on each side of the WAM-V, and a System Management Bus managed functions such as sending the remaining capacity and voltage to the CPU. The goal was to have a diesel generator charge the batteries and run the USV's load when the batteries depleted.

The continuous process of charging and discharging batteries will increase the range on different missions by at least 50 miles, and the approach will be scalable with the size of the diesel tank and the USV. Consequently, this will allow the system to rely on diesel fuel rather than the batteries. Within the range extender, several key components were needed, including an appropriate generator, smart batteries, and a battery manager. A 175-pound generator was chosen that has maximum output of 6500 watts and a continuous output of 6120 watts. This is double the power that our system requires for our predicted power use, and this provides flexibility to add different motors, batteries, or additional electronics in the future. The CPU onboard the WAM-V controls and manages the generator, thus deciding when the generator should be on or off.

The WAM-V is being outfitted with a variety of sensors to react to the world around it. Some sensors are crucial to basic USV functionality while others can be attached or removed to accommodate a specific mission. Sensors are either installed directly to the payload platform of the catamaran or attached to a gimbal-pole system that can lower into the water. An Inertial Measurement Unit (IMU) and Global Positioning System (GPS) are crucial sensors needed for most robotic platforms. A LIDAR is another crucial sensor used in mobile robotics platforms, both as a payload and navigation sensor in (for example) GPS-denied environments. The LIDAR reads depth information by rotating a laser about its horizontal axis and recording the depth of each point by the time spent for the light to travel and return back to the sensor. The LIDAR will be used to determine how far objects are from the WAM-V and help to map the environment while localizing itself within the map (Simultaneous Localization and Mapping [SLAM]like algorithms). A stereo camera will be used to classify, identify, and track objects in the near field. For example, it will be used to track the position of a UAV relative to the WAM-V to assist in landing on the WAM-V platform. The stereo camera can also be used to mosaic detailed 3-D maps of an environment, such as a harbor. Different underwater sonars will be used to provide complex mapping data under the surface, whereas LIDAR will provide a map of the environment above the surface. Our goal is to fuse these two maps for increased situational awareness.

\section{USER INTERFACE AND INTEGRATION OF SYSTEMS}

Using preexisting software and prototyping programs, a framework was developed to integrate, collaborate, control, and interact with this project. This incorporates a publish-subscribe framework for communications and control, as well as a mission control graphical user interface (GUI) that allows users to report and interact with the HAMMER system. Common controllers for multiple unmanned systems, programs to enable cooperative autonomy and autonomous command and control of UxS, and distributed control of unmanned systems using widgets, among other projects at varying levels of technical maturity, are already initiatives at SSC Pacific.

The U.S. Navy's focus is on developing CONOPS and performing autonomous controls research for unmanned systems. Distributed Control of Unmanned Systems Using Widgets, The Multi-Robot Operator Control Unit (MOCU), and the Intelligence Carry On Program (ICOP) are some examples of current initiatives at SSC Pacific that can be integrated into HAMMER. With these efforts in mind, a prototype has been created to model how the final GUI will function after all unmanned vehicles are interfaced together. 
The GUI is designed so that a user who may be unfamiliar with the UxV system will be able to easily learn the controls, leading to shortened training times. The GUI has been broken into two main pages: a GPS page and a data feed page. Each page will allow the user to switch to manual controls of the selected robot. The GPS page has been designed so that button selections are less obtrusive on the map than the traditional selection bar. This gives more screen area to the map and gives the user greater situational awareness. Buttons include map controls (e.g., zoom function, 3D, search bar), robot selection, robot controls (e.g., Sensor Feed, Status, Return to Vehicle, Shut Off) and a button to enter manual controls. By selecting a robot and clicking on the map, the user will be able to plot a waypoint to direct the robot to continue its mission to a specific location. The data page will allow the user to switch between the sensor feeds from each robot to allow easy monitoring of the robot's status. On the data feed page, the user will be able to monitor the stream of data detailing information such as the robot's heading, speed, and any other sensor information that is necessary for a status report. The manual controls were originally designed to give the user manual control over a robot using a joystick-type controller, with the goal of implementing on a touchscreen tablet, smart phone, or similar device.

\section{COOPERATING UNMANNED MARITIME VEHICLES}

Alongside the WAM-V surface vehicle, there are multiple other vehicles with this heterogeneous system. In order to cover all dimensions, there are accompanying underwater and aerial vehicles to augment the surface system and to provide extended services to the HAMMER project. Together, this system, as a whole, will use a fusion of sensor and user information to perform different missions in a variety of environments. The UAV, or unmanned aerial system (UAS), is comprised of an aerial vehicle, a payload, and onboard sensors and computer equipment. The current design of the UAS involves a helicopter vehicle with onboard autopilot and power system, as well as available space for a 10-pound payload.

The requirement for such a system is a 45 -minute to one-hour flight time, and capabilities to succeed in various weather and sea states. Currently, our research is focused on core tasks for the UAV, such as takeoff and landing on the USV. A single camera could then be used to perform stereolike vision since the distance between points on the landing deck are known. Since filters and optics exist with the capability to increase the effectiveness of a camera in varying weather and light conditions, a camera could be added that would meet the requirements for a UAV mission. Previously, imagery has been collected with a remotely-piloted hexcopter vehicle. In the future, other sensors, such as thermal, infrared, LiDAR, and ultraviolet cameras, will be tested for effectiveness in varying conditions.
Landing was the first focus, since it posed a more challenging problem that could also give insight on takeoff and other behaviors tied with the USV. Landing is characterized by: 1) GPS range calibration, 2) detection and tracking of the USV and landing platform, 3) sensor fusion of IMU and camera data from the UAV and USV, and 4) path planning to define an optimal and closely-tracked path for the landing descent. First, the UAV and the USV must get within range of each other using their GPS coordinates.

Once in range of each other using GPS, the next step utilizes the three-point algorithm that uses a single camera to perform depth calculations [5]. Some adaptations are made in our implementation: the three points are required to be orthogonal and aligned with the USV body axes, and all three points are on the same plane. The HAMMER system provides redundant systems for localization; the use of a single camera on the UAS or a combination of sensors on the USV can provide multiple, robust solutions for localization. This requires some sophisticated intelligence in UAS autonomy for path planning and computer vision.

Taking off from the USV is another capability required of this system. Since the USV and UAV will have a strong communications link and can work together before takeoff, this becomes a relatively straightforward task between the two systems. Data can be collected to inform the UAV of sea and air state, which can impose limitations on the UAV's takeoff envelope. Once the data collected indicates it suitable to fly, the UAV can start its motors with a downward force to minimize any lateral movement that might occur during unlatching. Different approaches are being studied for unlatching the UAV from the landing platform, such as latching mechanisms, tensioning and spooling tethers, electromagnetic solutions, and others.

\section{CONCLUSIONS}

With the propulsion, power management, and sensor systems of the USV, the landing and takeoff algorithms of the UAV, the future integration of a UUV, and its overarching communications framework, the HAMMER project is soon to achieve heterogeneous system autonomy. Several subsystems working together will minimize operator requirements and reduce the manpower necessary for system success.

Past successes of UxV integrations into the U.S. Navy Fleet are good examples to follow, most notably the introduction of the Fire Scout UAV. This system will operate together with the MH-60 helicopter detachment with the net result being no increase in manning. This is precisely the path UxVs deployed from naval ships must follow. In the future, we believe it will be necessary for the Navy to reduce manpower on Navy ships and cross-train Sailors still aboard Navy ships, all while increasing capabilities. This may even 
lead to a new CONOPS for unmanned systems deployed from Navy ships in which the operators are not located on the ship at all.

RADM William Leigher, USN (Ret.), former Director of Warfare Integration for Information Dominance, noted that the future of intelligence is "automated systems that can analyze and fuse enough intelligence information from multiple sources to begin to predict events [6]." Our goals with the HAMMER system encompass that-getting $80 \%$ of the way there without waiting the decade(s) it would take to reach $99 \%$ of the proposed capabilities. The HAMMER system is being developed as an autonomous system to be deployed for months at a time, with both storing and continuous data links.

UAVs like the Fire Scout could be used as a solid link between an LCS ship and some HAMMER system counterparts at sea, forming a multipurpose and extensive system to be used by the Navy for Information Dominance. Remaining challenges to overcome come in the form of GPS and satellite-denied environments, as well as the inherent limitations of communications partnered with large bandwidth sensors. Unmanned systems will need to gain the ability to perceive and understand the environment, a goal that will require significant research to enhance their current perception capabilities.
In the long term, these capabilities will eventually extend to the ability to learn from their environment and experiences, and to adapt appropriately in response. This is paramount to a system that can work on unpredictable and various missions, and can allow unmanned systems the ability to find the optimal solution for achieving their mission without a need to rely on constant human operator oversight and input. The HAMMER system is providing a modular testing platform to exercise these various missions. None of this can happen unless there is an optimal balance of autonomy and operator oversight and, when necessary, intervention.

\section{REFERENCES}

[1] M. Boot, War Made New: Technology, Warfare, and the Course of History 1500 to Today (New York: Gotham Books, 2006), pp. 318-351.

[2] B. Berkowitz, The New Face of War: How War Will Be Fought in the $21^{\text {st }}$ Century (New York: The Free Press, 2003).

[3] The Congressional Budget Office report, Costs of Military Pay and Benefits in the Defense Budget, November 14, 2012

[4] T.B. Curtin, J. G. Bellingham, J. Catipovic, and D. Webb "Autonomous oceanographic sampling networks," Oceanography, Vol. 6, pp. 86-94, 1993.

[5] P. Ghyzel, "Vision-based Navigation for Autonomous Landing of Unmanned Aerial Vehicles." Thesis. Naval Postgraduate School, 2000. at:

$<$ http://edocs.nps.edu/npspubs/scholarly/theses/2000/Sep/00Sep_Ghyzel .pdf $>$

[6] W. Matthews, "Keeping Pace," Seapower Magazine, December 2011. 